\title{
Robotic versus total laparoscopic radical hysterectomy with pelvic lymphadenectomy for the treatment of early cervical cancer
}

\author{
Jagdishwar G Goud, Kiranmai Gottapu*, Vikas Kumar MB, Arun Katari, Kaveri Shaw
}

Department of Surgical Oncology and Robotic Surgery, Krishna Institute of Medical Sciences, Secunderabad-500003, Andhra Pradesh, India

Received: 1 November 2013

Accepted: 13 November 2013

*Correspondence:

Dr. Kiranmai Gottapu,

E-mail: kiranmaigottapu@yahoo.com

(C) 2014 Goud JG et al. This is an open-access article distributed under the terms of the Creative Commons Attribution Non-Commercial License, which permits unrestricted non-commercial use, distribution, and reproduction in any medium, provided the original work is properly cited.

\begin{abstract}
Background: The aim of this study is to compare the safety, morbidity, intra operative, pathologic and postoperative outcomes of Robotic radical hysterectomy (RRH) to total laparoscopic radical hysterectomy (TLRH) in patients with early stage cervical cancer.

Methods: All the women with newly diagnosed invasive cervical cancer (stage IB1to IIA), who underwent TLRH or RRH with pelvic lymph node dissection at Krishna Institute of Medical Sciences from September 2011 to August 2013 were analysed.

Results: Twenty six patients underwent TLRH with pelvic lymphadenectomy and twenty six patients underwent RRH with pelvic lymphadenectomy. Age, tumor histology, stage, lymphovascular space involvement and nodal status are same for both the groups. No statistical differences were observed in operative time (174 vs. 158 min), estimated blood loss (160 vs. $110 \mathrm{ml}$ ), or hospital stay (3.1 vs. 2.8 days). Mean pelvic lymph node count was more in Robotic group. None of the robotic or laparoscopic procedures required conversion to laparotomy. All patients in both groups are alive and free of disease at the time of last follow up.

Conclusions: According to our experience, robotic radical hysterectomy appears to be safe and effective therapeutic procedure for managing early-stage cervical cancer without significant differences when compared to TLRH, with respect to operative time, blood loss, hospital stay. Regarding the oncological outcome, Robotic radical hysterectomy is superior in terms of number of lymph nodes and parametrial bulk; although multicenter randomized clinical trials with longer follow-up are necessary to evaluate the overall oncologic outcome.
\end{abstract}

Keywords: Laparoscopic, Robotic, Radical hysterectomy, Pelvic lymphadenectomy, Cervical cancer

\section{INTRODUCTION}

Open radical hysterectomy has been the standard treatment for early stage cervical cancer for decades. The first total laparoscopic radical hysterectomy (TLRH) with pelvic and paraaortic lymphadenectomy was performed by Nezhat et al. in $1989 .{ }^{1,2}$ Since then, TLRH with pelvic or paraaortic lymph node dissection has gained acceptance as a feasible alternative to an open radical hysterectomy. Recent advances in laparoscopic instrumentation, however, have made it possible to safely perform radical hysterectomy laparoscopically.

Despite the advantages of conventional laparoscopy over laparotomy (shorter hospitalization, faster bowel function recovery, less postoperative pain, decreased overall cost), it has its own drawbacks like uncomfortable position at the operating table, flat, 2-dimensional image, nonarticulating instruments with an ergonomically inadequate handle design and with significant learning 
curve mostly due to the counterintuitive nature of the operation.

Recently, computer enhanced technology (robotics) has been introduced into laparoscopic surgical practice. The advantages offered by this new technology include a 3 dimensional magnified field, tremor filtration, and 7 degrees of instrument mobility inside the body, thus significantly reducing the ergonomic problems associated with the conventional laparoscopic approach. There is convincing evidence that the intuitive nature of the robotic system provides an additional advantage in terms of the learning curve.

Clinical applications for robotic systems have been evolving rapidly and are now used widely in various surgical fields. In this study, we conducted a comparative analysis of our data from early cervical cancer patients who underwent TLRH versus those who had RRH with respect to intraoperative, pathologic, and postoperative outcomes.

\section{Aim}

To compare the safety, morbidity, intra operative, pathologic and postoperative outcomes of robotic radical hysterectomy (RRH) to total laparoscopic radical hysterectomy (TLRH) in patients with early stage cervical cancer.

\section{METHODS}

Medical All the women with newly diagnosed invasive cervical cancer (stage IB1 to IIA), who underwent TLRH or RRH with pelvic lymph node dissection at Krishna Institute of Medical Sciences from September 2011 to August 2013 were analysed.

\section{Study design}

A prospective nonrandomized analysis of all cases of Radical Hysterectomy, either laparoscopic or robotic approach performed for cervical cancer at Krishna Institute of Medical Sciences, Secunderabad. All patients were appropriately counselled and written informed consent was obtained. Institutional Review Board approval was taken.

\section{Data collection}

All patients were staged according to the International Federation of Gynecology and Obstetrics (FIGO) criteria. All patients had a computerized tomography (CT) scan of the abdomen and pelvis done preoperatively to evaluate lymph node status and potential extra pelvic and extra abdominal disease. Clinical data were analysed by a review of patients' medical records and operation reports and histopathology reports.

\section{Surgical technique- robotic radical hysterectomy}

After appropriate preoperative counselling and written informed consent, a standard outpatient mechanical bowel preparation and perioperative prophylactic antibiotics were given. The procedure was performed with the patient under general anesthesia in the dorsal lithotomy position with adjustable Allen stirrups and lower extremity compression devices for deep venous thrombosis prophylaxis. Betadine solution was applied topically, and sterile drapes were placed in the usual sterile fashion. A Foley catheter was placed into the bladder before the procedure was started; the catheter was drained by gravity for the duration of the surgery.

Traditional diagnostic laparoscopy was performed first to assess for feasibility of the intended procedure, as well as to detect intraabdominal metastatic disease. The procedure was terminated if metastatic disease was detected, and confirmed by frozen section. If found feasible, we proceed for radical hysterectomy. A standard 12-mm trocar, placed at the umbilicus was used for camera placement, 2 working robotic arms were attached to $8-\mathrm{mm}$ reusable trocars placed bilaterally, one $8 \mathrm{~mm}$ trocar for 3rd arm in left or right iliac fossa and ancillary 10-mm trocar placed in the left or right upper quadrant (Figure1). The robotic ports were placed $1 \mathrm{~cm}$ to $2 \mathrm{~cm}$ below and $8 \mathrm{~cm}$ to $10 \mathrm{~cm}$ lateral to the camera port, so as to enable optimal movement of the robotic arm and to minimize the risk of collision (Figure 2).

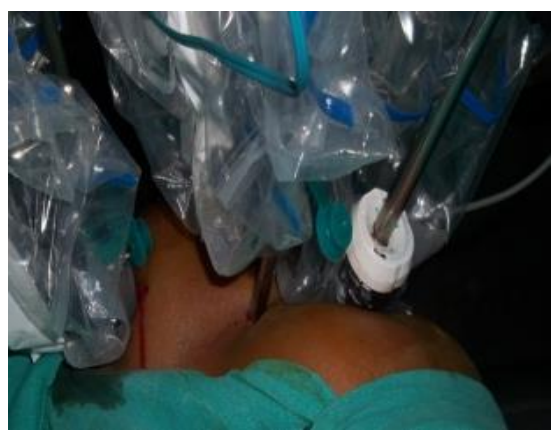

Figure 1: Trocar placement for robotic radical hysterectomy.

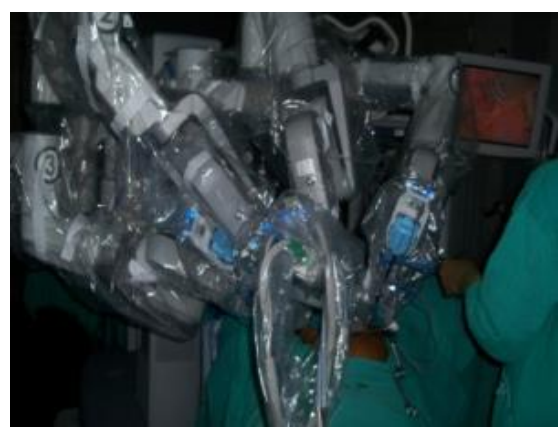

Figure 2: After docking the robot. 
The whole procedure is performed using the robotic monopolar electrosurgical scissors placed through the 1 st arm, the fenestrated bipolar forceps placed in $2^{\text {nd }}$ arm and prograsp in $3^{\text {rd }}$ arm. Conventional instruments used are the suction irrigator pump, grasping forceps and clip applicator as needed.

Adhesions were lysed first to restore normal anatomy, and the undersurfaces of the diaphragm, liver, gallbladder, stomach, omentum, and large and small bowel were examined visually, when possible. The paraaortic lymph nodes were inspected, followed by the pelvic lymph nodes. Proceeding with a radical hysterectomy requires that 6 avascular pelvic spaces be developed and that the bladder and rectum be mobilized.

After round ligaments on either side of the uterus were desiccated and cut with the monopolar scissors, the anterior leaf of the broad ligament was opened bilaterally (Figure 3). The bladder flap was developed using both blunt and sharp dissection. The bladder was gradually dissected away from the cervix and vagina. The uterus is pushed cephalad into the abdominal cavity to facilitate visualization (Figure 4).

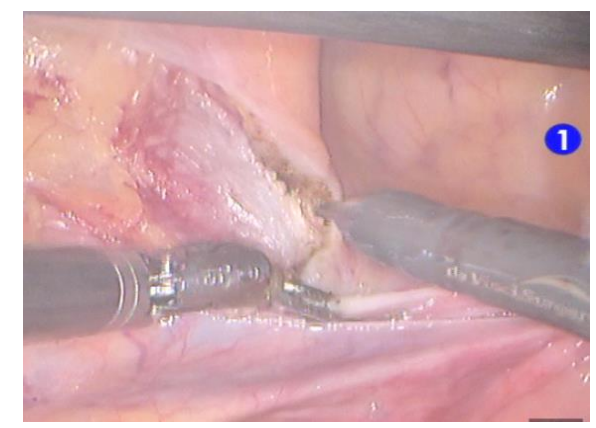

Figure 3: Round ligament dessicated and cut.

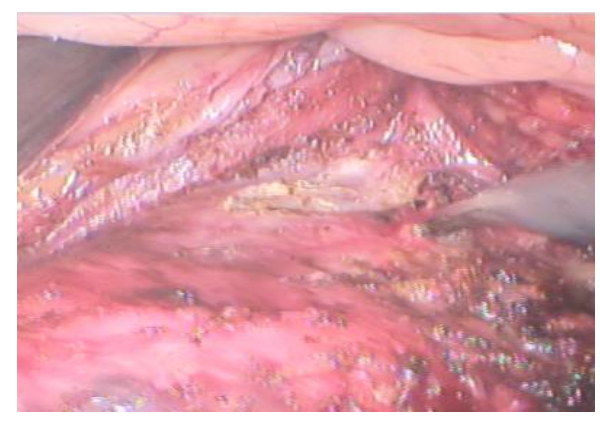

Figure 4: Development of the vesicovaginal space. The uterus is pushed cephalad into the abdominal cavity to facilitate visualization.

Then we proceed with pelvic lymph node dissection. Pelvic lymphadenectomy involves removal of the lymph node packets with surrounding lymphoareolar tissue from the common iliac vessels and external iliac vessels down to the level of the deep circumflex iliac veins (Figure 5). The obturator nerve was identified, and the obturator fossa nodes and the hypogastric lymph nodes were completely removed enblock (Figure 6). The lymph node packets on both sides are taken into self-made pouches separately with different color code. At this point, the medial umbilical ligament was suspended with upward tension, and the origin of the uterine artery from the hypogastric artery was identified and clipped (Figure 7). The uterine artery was desiccated and divided at its origin with bipolar forceps and monopolar scissors as (Figure 8). The uterine vein was likewise identified, desiccated, and cut.

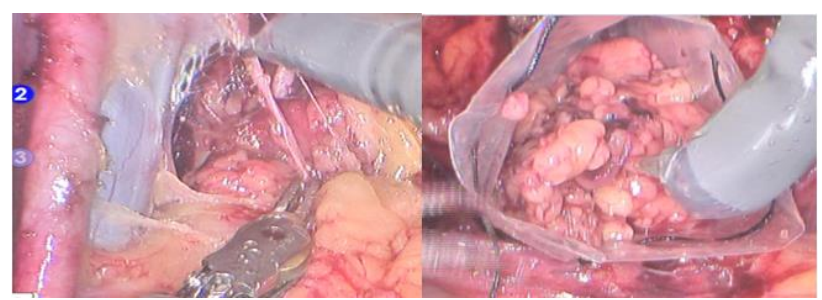

Figure 5: Pelvic lymphadenectomy. Lymph node packets are removed from the left common external iliac artery and vein and obturator fossa.

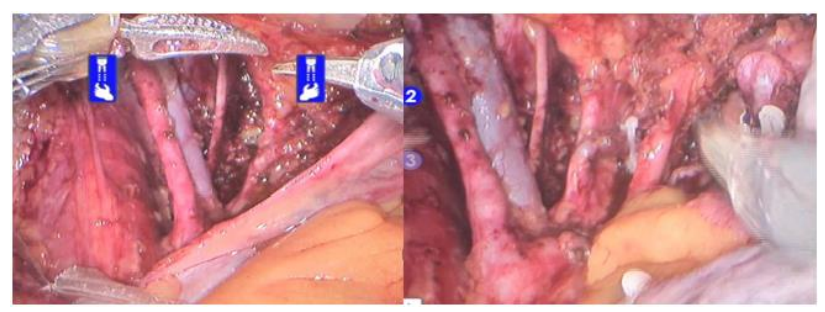

Figure 6: Obturator nerve and obturator fossa and pelvic sidewall.

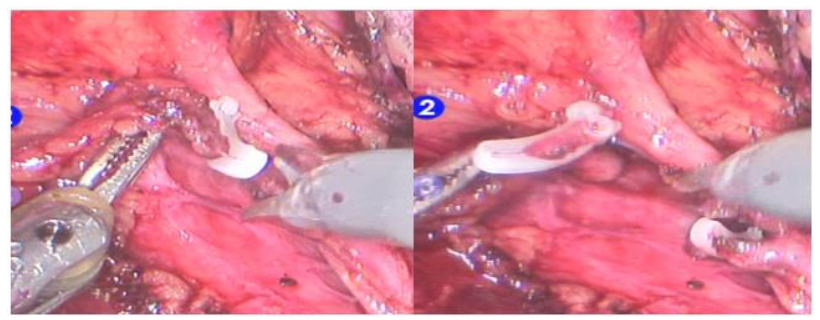

Figure 7: The uterine artery is identified and dissected from the point of its origin at the hypogastric artery, clipped and cut.

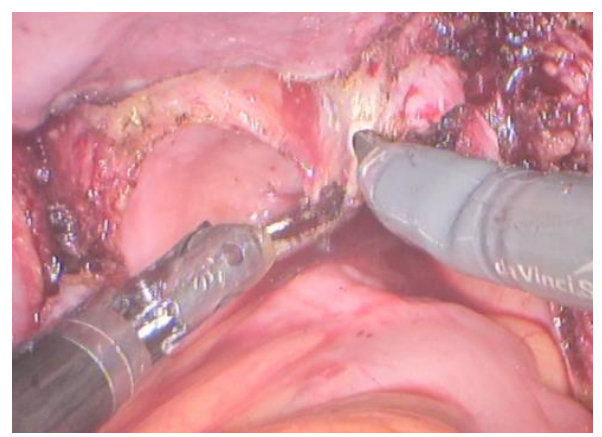

Figure 8: Development of the rectovaginal space. 
The peritoneum between the uterosacral ligaments is incised by using monopolar scissors; the rectum can then be brought down gently away from the vagina (Figure 8).

The uterine vessels were placed on medial tension, and the ureter was unroofed using the curved tip of the monopolar scissor out of the tunnel (Figure 9), and then the surrounding tissues were coagulated and divided. The uterosacral ligaments, cardinal ligaments, and a portion of the paracolpos were then divided with the bipolar forceps and scissors, enabling complete mobilization of the uterus (Figure 10). A circumferential incision was made into the vagina using monopolar scissors to ensure an adequate margin (Figure 11). The infundibulopelvic ligament was isolated, clipped, desiccated and divided using the bipolar forceps and scissors (Figure12).

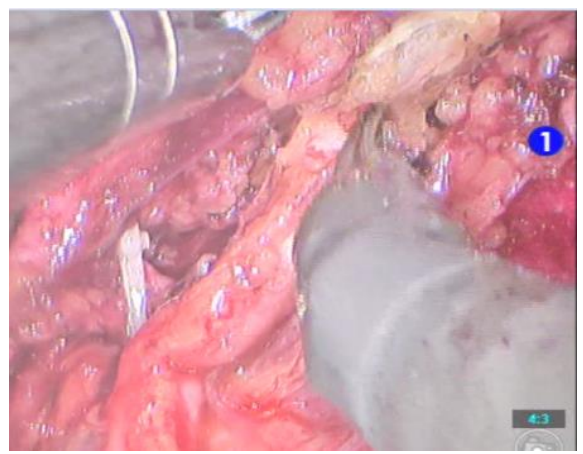

Figure 9: Unroofing of the right ureter using monopolar scissors.

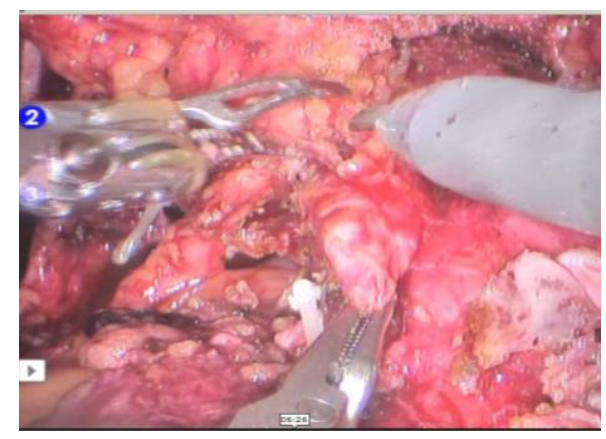

Figure 10: Dissection of parametrium.

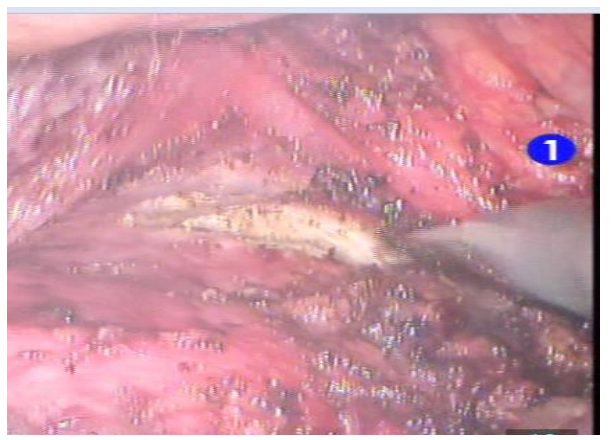

Figure 11: Opening the vault.

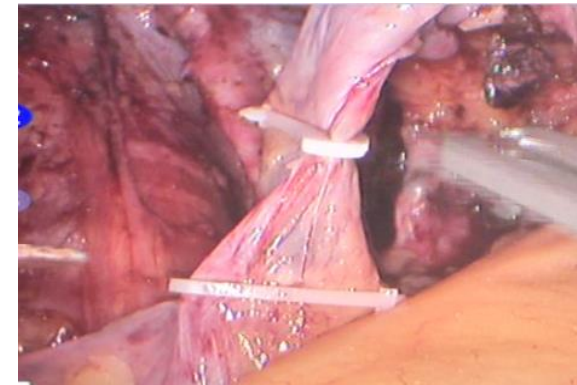

Figure 12: Clipping the infundibulopelvic ligament.

The uterus was separated completely from the vagina and removed while attached to the uterine manipulator. The specimen removal was done vaginally. The vaginal cuff was closed with continuous running 0 Vicryl suture tied intracorporeally (Figure 13).

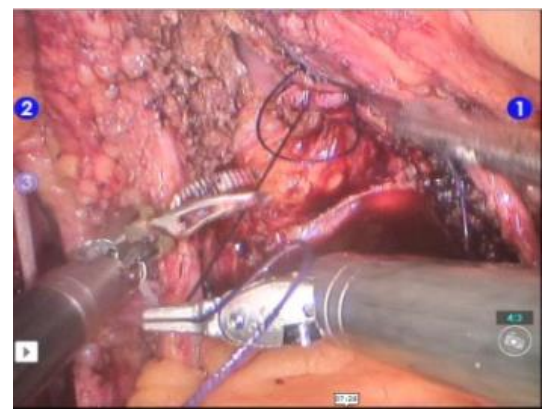

Figure 13: Vaginal cuff closure with intracorporeal tying.

After removal of the specimen and closure of the vaginal cuff, the pelvic cavity was thoroughly evaluated. Both the pelvic and abdominal cavities were irrigated copiously with normal saline and hemostasis ensured. Upon completion of the procedure, the da Vinci system was undocked, all of the instruments were removed, and the trocar sites were closed; rectus with prolene and skin with 3-0 monocryl in a subcuticular fashion.

\section{Statistical analysis}

Comparative analysis was performed using SPSS. The outcomes from the laparoscopic radical and roboticassisted groups were compared using the chi-square test for categorical variables and 2 sample Student $\mathrm{t}$ tests for continuous variables. $\mathrm{P}<0.05$ was considered significant in all cases.

\section{RESULTS}

A total of 52 patients met our inclusion criteria and had either TLRH or RRH with pelvic lymphadenectomy performed. The patient groups were similar with respect to age with mean age of 54.8 yrs (39-66) in RRH and $52.6 y r s$ (38-68) in TLRH. There were no differences in clinical tumor characteristics, such as stage, histology, 
and lymph-vascular space involvement between the two groups.

The mean operative time, estimated blood loss, and length of postoperative stay were similar between the two patient groups $(\mathrm{P}>0.05)$.

Duration of surgery was defined from the time of skin incision to the closure of the skin incision. Robotic docking time was recorded as the time to attach the robotic arms to the trocars and insertion of robotic instruments (Table 1). Blood loss was measured as a sum of suctioned fluids and weighed sponges. The mean estimated blood loss in RRH group is $110 \mathrm{ml}$ compared to $160 \mathrm{ml}$ in TLRH group (Table 2). Duration of hospital stay in both the groups is same between 2 to 5 days (Table 3).

Table 1: Duration of surgery.

\begin{tabular}{|lll|}
\hline & RRH $(\mathbf{n = 2 6})$ & TLRH $(\mathbf{n = 2 6})$ \\
\hline Mean duration of & 158 & 174 \\
surgery (minutes) & $(140-190)$ & $(150-210)$ \\
\hline
\end{tabular}

The mean duration of surgery in RRH group is $158 \mathrm{mts}$ compared to $174 \mathrm{mts}$ in TLRH group. The average docking time is 10 minutes.

Table 2: Estimated blood loss.

\begin{tabular}{|lll|}
\hline & RRH $(\mathbf{n = 2 6})$ & TLRH $(\mathbf{n = 2 6})$ \\
\hline Mean estimated & $110 \mathrm{ml}$ & $160 \mathrm{ml}$ \\
blood loss $(\mathrm{ml})$ & $(50-300)$ & $(80-400)$ \\
\hline
\end{tabular}

The mean estimated blood loss in RRH group is $110 \mathrm{ml}$ compared to $160 \mathrm{ml}$ in TLRH group.

Table 3: Hospital stay.

\begin{tabular}{|lll|}
\hline & RRH $(\mathbf{n = 2 6})$ & TLRH $(\mathbf{n = 2 6})$ \\
\hline Mean length of & 2.875 & 3.1 \\
hospital stay (days) & $(2-5)$ & $(2-5)$ \\
\hline
\end{tabular}

Duration of hospital stay is almost same in both the groups.

\section{Intraoperative \& postoperative complications}

None of the patients in either group had intra operative complication or required conversion to laparotomy. Postoperative complications in the RRH group included one case of postoperative ileus and one case of prolonged urinary retention. The TLRH group had complications like one case of deep vein thrombosis, one case each of ileus and prolonged urinary retention (Table 4).

\section{Pelvic lymph node count}

The mean yield of the pelvic lymph nodes was 27 in the RRH group and 20 in the TLRH group with significant $P$ value of 0.0318 (Table 5).
Table 4: Postoperative complications.

\begin{tabular}{|lll|}
\hline & RRH $(\mathbf{n = 2 6})$ & TLRH $(\mathbf{n = 2 6 )}$ \\
\hline Ileus & 1 & 1 \\
\hline DVT & 0 & 1 \\
\hline Urinary retention & 1 & 1 \\
\hline Total & 2 & 3 \\
\hline
\end{tabular}

Table 5: The mean yield of the pelvic lymph nodes.

\begin{tabular}{|l|l|l|}
\hline & RRH $(\mathbf{n = 2 6})$ & TLRH $(\mathbf{n = 2 6})$ \\
\hline $\begin{array}{l}\text { Mean total number } \\
\text { of pelvic nodes }(\mathrm{n})\end{array}$ & 27 & 20 \\
\hline
\end{tabular}

The mean yield of the pelvic lymph nodes as per the histopathology report was 27 in the RRH group and 20 in the TLRH group with significant $P$ value of 0.0318

\section{Follow up}

There were no recurrences in either group till now and in still follow up. All patients in both groups are alive and free of disease at the time of last follow up.

\section{DISCUSSION}

Several recent publications strongly demonstrated that computer-assisted surgical approaches are becoming increasingly feasible. Nezhat ${ }^{3}$ and colleagues and Koh and colleagues ${ }^{4}$ reported their experiences performing various advanced gynecologic procedures using the current generation of the da Vinci system. The largest experience using robotic systems for the surgical treatment of gynecologic cancers was reported by $\mathrm{J}$. Magrina of the Mayo Clinic (Scottsdale, AZ). ${ }^{5}$ It comprised 142 patients treated surgically with the da Vinci robotic system for various primary and recurrent gynecologic malignancies. The lymph node count was 27.9, with no intraoperative or postoperative complications. The authors concluded that robotic surgery is preferable to conventional laparoscopy for gynecologic oncology procedures. Boggess reported ${ }^{6}$ similar data after performing RRH at the University of North Carolina. The author performed $13 \mathrm{RRH}$ procedures that were compared with 48 historic abdominal radical hysterectomies. Lymph node yield was significantly higher in the robotic group (33 vs. 22).

In fact, our evidence, as well as the evidence of others, supports robotic surgery as a more attractive option, both for the surgeon and the patient.

Abdominal radical hysterectomy continues to be the most common surgical approach in treatment of an early stage carcinoma of the cervix. The role of laparoscopy in this setting is to offer all of the benefits of a minimally invasive approach, while maintaining the excellent oncological outcomes of an open approach. TLRH is one of the most challenging laparoscopic procedures in gynecologic oncology, requiring significant technical 
expertise and experience. Because this is a relatively new technique, the number of cases required to obtain proficiency is not known. As more centers perform these procedures, report their experiences and the technique itself is developed, standardized, and taught systematically, we will better understand the learning curve required for both TLRH and RRH.

As the number of early cervical cancer cases is decreasing, fast acquisition of advanced endoscopic skills is paramount. Therefore, the robotic interface, which allows for significant shortening of the learning curve, may make a minimally invasive approach possible even in centers with very few cases of early cervical cancer.

The robotic systems have their own drawbacks; most commonly mentioned are the absence of tensile feedback, the complexity of the system, the size of the system, and the cost. Robotic technology is developing rapidly, and new instruments, smaller arms, the addition of a fourth arm and tactile feedback is already becoming available. Currently, operations performed with a robot are expensive, but the widespread use of this technology, combined with the shorter hospital stay, hopefully, will lead to an overall, and substantial, decrease in cost

\section{CONCLUSIONS}

According to our experience, robotic radical hysterectomy appears to be safe and effective therapeutic procedure for managing early-stage cervical cancer without significant differences when compared to TLRH, with respect to operative time, blood loss, hospital stay. Regarding the oncological outcome, Robotic radical hysterectomy is superior in terms of number of lymph nodes and parametrial bulk; although multicenter randomized clinical trials with longer follow-up are necessary to evaluate the overall oncologic outcome.

Funding: No funding sources

Conflict of interest: None declared

Ethical approval: Not required

\section{REFERENCES}

1. Nezhat CR, Burrell MO, Nezhat FR, Benigno BB, Welander CE. Laparoscopic radical hysterectomy with paraaortic and pelvic node dissection. Am J Obstet Gynecol. 1992;166:864-5.

2. Nezhat C. Laparoscopic radical hysterectomy with paraaortic and pelvic lymph node dissection? Am J Obstet Gynecol. 1993;168:1644-5.

3. Nezhat C, Saberi NS, Shahmohamady B, Nezhat F. Robotic-assisted laparoscopy in gynecological surgery. JSLS. 2006; 10:317-20.

4. Kho RM, Hilger WS, Hentz JG, Magtibay PM, Magrina JF. Robotic hysterectomy: technique and initial outcomes. Am J Obstet Gynecol. 2007;197:113e1-4.

5. Magrina JF. Robotic surgery in gynecology. Eur $\mathbf{J}$ Gynaecol Oncol. 2007;28:77-82.

6. Boggess J. Robotic surgery in gynecologic oncology: evolution of a new surgical paradigm. J Robotic Surg. 2007;1:31-7.

DOI: $10.5455 / 2320-1770$. ijrcog20140306

Cite this article as: Goud JG, Gottapu K, Kumar

VMB, Katari A, Shaw K. Robotic versus total laparoscopic radical hysterectomy with pelvic lymphadenectomy for the treatment of early cervical cancer. Int J Reprod Contracept Obstet Gynecol 2014;3:34-9. 